\title{
Phycoerythrocyanin and Phycoerythrin: Properties and Occurrence in Cyanobacteria
}

\author{
By DONALD A. BRYANT $\dagger$ \\ Unité de Physiologie Microbienne, Département de Biochimie et Génétique Moléculaire, \\ C.N.R.S.-E.R.A. 398, Institut Pasteur, 28, rue du Docteur Roux, F-75015 Paris, France
}

(Received 13 October 1981)

\begin{abstract}
A number of cyanobacterial phycoerythrocyanins were isolated and characterized with respect to spectroscopic properties and chromophore content. All were similar to the phycoerythrocyanin of Anabaena PCC 6411 and cross-reacted strongly with an antiserum prepared against that protein. The synthesis of phycoerythrocyanin was not controlled by a complementary chromatic adaptation mechanism in nine strains tested, but its synthesis appeared to be affected by light intensity. A survey of 240 strains of cyanobacteria revealed that no strain synthesized both phycoerythrocyanin and phycoerythrin and that phycoerythrocyanin was largely confined to those strains which can form heterocysts. Phycoerythrocyanin may be a taxonomically useful marker for certain strain clusters of this cyanobacterial grouping. Spectroscopic properties and chromophore compositions of several cyanobacterial phycoerythrins were also determined. At present, the ability to synthesize phycoerythrin and the capacity to exhibit complementary chromatic adaptation responses are widely distributed traits of limited taxonomic usefulness.
\end{abstract}

\section{INTRODUCTION}

Phycobiliproteins are the major photosynthetic accessory pigments of cyanobacteria and red algae. These proteins are assembled into supramolecular complexes, phycobilisomes, which form regular arrays on the stromal surfaces of the thylakoid membranes of both groups of organisms (Gantt, 1980). On the basis of their long-wavelength visible absorption properties, the phycobiliproteins have been classified into several groups (Table 1).

Spectroscopic studies performed with isolated phycobilisomes have demonstrated that the phycobiliproteins constitute an energy transfer chain (Ley et al., 1977; Searle et al., 1978):

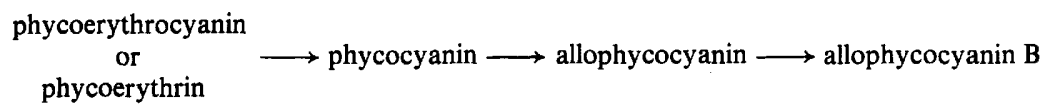

Within the cell, light energy trapped by the phycobiliproteins is transferred to chlorophyll $a$ with an efficiency approaching $100 \%$ (Porter et al., 1978).

Phycocyanin and allophycocyanin are universally present in cyanobacteria, whilst allophycocyanin B apparently occurs in most cyanobacteria (Glazer \& Bryant, 1975; Ley et al., 1977) but was not detected in the unicellular cyanobacterium Gloeobacter violaceus (Bryant et al., 1981). Phycoerythrins are widely distributed among all taxonomic groupings and form the most spectroscopically variable class of phycobiliproteins (Stanier \& Cohen-Bazire, 1977; Table 1). Many, but not all, cyanobacteria containing phycoerythrin undergo an adaptive phenomenon known as complementary chromatic adaptation (Bogorad,

† Present address: Department of Microbiology, Cell Biology, Biochemistry and Biophysics, 101 South Frear Building, The Pennsylvania State University, University Park, PA 16801, U.S.A. 


\section{Table 1. Distribution and properties of biliproteins}

Data were taken from Glazer (1977), Muckle \& Rüdiger (1977), Glazer \& Hixson (1977) and Nies \& Wehrmeyer (1980). C and $R$ indicate that the protein occurred in cyanobacteria and red algae, respectively. The exact positions of the absorption and fluorescence emission maxima vary depending upon the aggregation state of the protein and upon the organism which serves as the source of the protein. Abbreviations for the phycobilin chromophores are: $\mathrm{PCB}$, phycocyanobilin; PEB, phycoerythrobilin; PXB, phycobiliviolin-type chromophore of undetermined structure.

\begin{tabular}{|c|c|c|c|c|c|}
\hline Biliprotein & Distribution & $\begin{array}{l}\text { Visible absorption } \\
\text { maxima }(\mathrm{nm})^{*}\end{array}$ & $\begin{array}{l}\text { Fluorescence } \\
\text { emission } \\
\text { maximum (nm) }\end{array}$ & $\begin{array}{c}\text { Subunit structure } \\
\text { of stable } \\
\text { assembly forms }\end{array}$ & $\begin{array}{l}\text { Chromophore } \\
\text { content of the } \\
\text { promoter }(\alpha \beta)\end{array}$ \\
\hline ycocyanin B & $\mathrm{C}, \mathrm{R}$ & $671>618$ & 675 & $(\alpha \beta)_{3}$ & 2 PCB \\
\hline ycocyanin & $\mathrm{C}, \mathrm{R}$ & 650 & 660 & $(\alpha \beta)_{3}$ & 2 PCB \\
\hline cocyanin & $\mathrm{C}, \mathrm{R}$ & 620 & 640 & $(\alpha \beta)_{3} ;(\alpha \beta)_{6}$ & 3 PCB \\
\hline cocyanin & $\mathbf{R}$ & $617>555$ & 636 & $(\alpha \beta)_{3} ;(\alpha \beta)_{6}$ & 2 РСB; 1 PEB \\
\hline erythrocyanin & C & $570>595(\mathrm{~s})$ & 625 & $(\alpha \beta)_{3} ;(\alpha \beta)_{6}$ & 2 РCB; 1 PXB \\
\hline coerythrin & $\mathrm{C}$ & 560 & 577 & $(\alpha \beta)_{3} ;(\alpha \beta)_{6}$ & 5-6 PEB \\
\hline coerythrin & $\mathbf{R}$ & $545>563(\mathrm{~s})$ & 570 & $(\alpha \beta)_{\mathrm{n}}$ & 6 PEB \\
\hline coerythrin & $\mathbf{R}$ & $545>563>498(s)$ & 575 & $(\alpha \beta)_{6} \gamma$ & $6 \mathrm{PEB} \dagger$ \\
\hline ycoerythrin & $\mathrm{C}, \mathrm{R}$ & $565>540>498$ & 578 & $(\alpha \beta)_{6} \gamma$ & $\mathrm{ND} \ddagger$ \\
\hline
\end{tabular}

ND, Not determined.

* (s), shoulder.

† The $\gamma$ subunit of Porphyridium cruentum B-phycoerythrin carries two phycoerythrobilins and two phycourobilin chromophores.

$\ddagger$ Cryptonemia obovata R-phycoerythrin carries 31 phycoerythrobilin and 9 phycourobilin chromophores $\operatorname{per}(\alpha \beta)_{6} \gamma$.

1975). Tandeau de Marsac (1977) demonstrated that two responses can occur: either phycoerythrin synthesis alone, or phycoerythrin and phycocyanin synthesis is controlled in response to the light wavelength incident during growth.

Phycoerythrocyanin is a cyanobacterial phycobiliprotein functionally similar to phycoerythrin but bearing some structural similarities to phycocyanin (Bryant et al., 1976, 1978; Fueglistaller et al,, 1980). The absorption properties of phycoerythrocyanin can effectively extend to shorter wavelengths the light-harvesting range of organisms containing this biliprotein (Table 1). In confirmation of its role as a light-harvesting accessory pigment, phycoerythrocyanin has been shown to be present in the phycobilisomes of Anabaena PCC 6411 (Bryant et al., 1976) and Fischerella PCC 7603 (Nies \& Wehrmeyer, 1980) [Fischerella PCC 7603 = Mastigocladus laminosus Cohn (Rippka et al., 1979)].

Although no systematic search had been made, it was suggested that phycoerythrocyanin had a limited distribution among the cyanobacteria (Bryant et al., 1976). This report describes the results of screening 240 strains of cyanobacteria for the presence of phycoerythrocyanin and/or phycoerythrin. The spectroscopic properties of several phycoerythrocyanins and phycoerythrins are described. The survey of Tandeau de Marsac (1977), concerning the distribution and pattern of chromatic adaptability among cyanobacteria which synthesize phycoerythrin, was extended to include recently isolated strains and some strains containing phycoerythrocyanin. The taxonomic significance of the occurrence of phycoerythrocyanin and phycoerythrin, and of chromatic adaptation, among cyanobacteria is discussed. Some preliminary results of this screening were reported by Rippka et al. (1979).

\section{METHODS}

Strains and culture conditions. Axenic strains of cyanobacteria were obtained from the Pasteur Culture Collection (PCC; Rippka et al., 1979; Rippka \& Stanier, 1979). A few strains contained unpigmented bacterial contaminants (Tables 2, 3 and 4). Unless specified, all cultures were grown photoautotrophically at 1.0 to $2.0 \times 10^{2}$ 
erg $\mathrm{cm}^{-2} \mathrm{~s}^{-1}$ under white fluorescent lamps (Osram Universal). Freshwater strains were grown in medium BG-11 (Stanier et al., 1971) and strains of marine origin were grown in medium MN or ASN-III (Rippka et al., 1979).

Materials. Electrophoresis-grade acrylamide and bisacrylamide were purchased from Serva Feinbiochemica, Heidelberg, F.R.G., and from Eastman Organic Chemicals, respectively; ampholytes (40\% w/v; Bio-Lyte) from Bio-Rad; streptomycin sulphate from Merck; and pre-swollen Whatman microgranular DEAE-cellulose DE-52 from Whatman Biochemicals. All other chemicals were reagent grade.

Chromatic adaptation studies. Comparative studies of the effect of light wavelength on pigmentation were performed as described by Tandeau de Marsac (1977). Culture conditions, light intensities, pigment extractions and the optical properties of the filters used were the same as previously described (Tandeau de Marsac, 1977).

Spectroscopic measurements. Absorption spectra were measured with a Cary Model 17 recording spectrophotometer. Phycobiliprotein concentrations were estimated spectrophotometrically using previously published absorption coefficients (Bryant et al., 1976, 1979).

Polyacrylamide gel electrophoresis and isoelectric focusing. Electrophoresis at $\mathrm{pH} 7.5$ on $5 \%(\mathrm{w} / \mathrm{v})$ polyacrylamide gels $(0.6 \times 10$ to $12 \mathrm{~cm})$ was performed as described by Cohen-Bazire et al. (1977). Isoelectric focusing was performed on photopolymerized $7.0 \%$ (w/v) polyacrylamide gels in the $\mathrm{pH}$ range 4.0 to 6.0 , according to the procedure of Bio-Rad Laboratories (Technical Bulletin 1030, April, 1975). For some experiments, samples were mixed with the acrylamide solution before photopolymerization.

Immunological studies. Rabbit antiserum against Anabaena PCC 6411 phycoerythrocyanin and Synechocystis PCC 6808 phycoerythrin were obtained as described by Glazer et al. (1971) and Bryant et al. (1976). Immunodiffusion experiments were performed as described by Glazer \& Bryant (1975).

Preparation of cell extracts. All buffers contained $2 \mathrm{~mm}$-azide and, unless specified, all operations were performed at room temperature.

Cells were washed and suspended in $10 \mathrm{~mm}$-sodium acetate buffer $\mathrm{pH} 5.5$ (about 0.3 to $0.5 \mathrm{~g}$ wet wt cells ml $\mathrm{m}^{-1}$ ) and disrupted by two passages through an Aminco French pressure cell operated at $140 \mathrm{MPa}$ at $4^{\circ} \mathrm{C}$. Cell extracts were adjusted to contain $1 \%(\mathrm{w} / \mathrm{v})$ streptomycin sulphate. The suspensions were incubated $15 \mathrm{~min}$ at $0{ }^{\circ} \mathrm{C}$ and precipitated membranes removed by centrifugation at $27000 \mathrm{~g}$ for $15 \mathrm{~min}$. The supernatants were decanted and the pellets discarded. The supernatant was applied to a small column $(1.5 \times 3.0 \mathrm{~cm})$ of DEAE-cellulose which had been pre-equilibrated with $10 \mathrm{mM}$-sodium acetate buffer $\mathrm{pH} 5.5$. After washing the column with two column volumes of starting buffer, the phycobiliproteins were frontally eluted with $0.30 \mathrm{M}$-sodium acetate buffer $\mathrm{pH} 5 \cdot 5$. Solid ammonium sulphate was added to the eluate to $65 \%$ of saturation. The precipitates were stored at $4{ }^{\circ} \mathrm{C}$ for no more than 1 month before analysis.

Ammonium sulphate precipitates were collected by centrifugation for $10 \mathrm{~min}$ at $27000 \mathrm{~g}$, resuspended in small

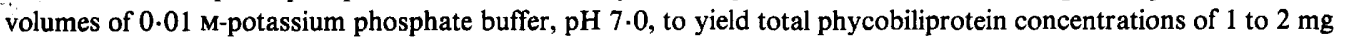
$\mathrm{ml}^{-1}$, and exhaustively dialysed against the same buffer. The resulting protein solutions were suitable for analysis by isoelectric focusing or polyacrylamide gel electrophoresis at $\mathrm{pH} 7.5$.

Purification and the detection of phycoerythrocyanin and phycoerythrin. Preparative polyacrylamide gel electrophoresis and preparative isoelectric focusing were effective methods for the purification of small amounts of phycoerythrocyanin and phycoerythrin. Larger amounts of Chroococcidiopsis PCC 7203 and Fischerella PCC 7603 phycoerythrocyanins were purified by chromatography of cell extracts on DEAE-cellulose and hydroxyapatite as previously described (Bryant et al., 1976).

Isoelectric focusing allowed rapid and sensitive screening for the presence of phycoerythrocyanin and/or phycoerythrin. Samples (100 to $400 \mu \mathrm{g}$ phycobiliprotein) of cell extract were subjected to isoelectric focusing in the $\mathrm{pH}$ range 4.0 to 6.0 . Phycoerythrocyanin was easily distinguishable from phycoerythrin by several criteria. (1) Phycoerythrocyanin is reddish-purple in colour, whereas phycoerythrins are reddish-orange. (2) When excited by long-wavelength u.v. illumination, phycoerythrocyanin fluorescence is reddish-orange (approx. 610 to $625 \mathrm{~mm}$; Bryant et al., 1976; Nies \& Wehrmeyer, 1980) whereas phycoerythrins fluoresce golden-yellow (approx. 575 to $580 \mathrm{~nm}$; Glazer, 1977). (3) The isoelectric points of phycoerythrocyanins detected fell into a narrow pH range of about 5.2 to 5.6, as previously reported for two phycoerythrocyanins (Bryant et al., 1976); phycoerythrins generally had more acidic isoelectric points $(\mathrm{pH} 4.5$ to 5.1$)$. (4) The reddish-coloured a subunit of phycoerythrocyanin was also frequently detected on the gels; this more basic subunit (pI approx. 6.0) was also a useful distinguishing feature. No phycoerythrins or phycoerythrin subunits were observed with isoelectric points greater than $\mathrm{pH} 5 \cdot 5$.

Further confirmation of the presence of phycoerythrocyanin or phycoerythrin was obtained by excising the coloured bands, eluting the proteins from the acrylamide gel and examining the absorption spectra of the eluted proteins. Eluted proteins were also examined with antibodies specific for phycoerythrocyanin and phycoerythrin. Antisera prepared against phycoerythrocyanins do not cross-react with phycoerythrins, and similarly, antisera prepared against phycoerythrins do not cross-react with phycoerythrocyanins (Bryant et al., 1976; Bryant, 1977). For this study all identifications of phycoerythrocyanin-positive strains were confirmed by double-diffusion studies against rabbit antiserum to Anabaena PCC 6411 phycoerythrocyanin. Strong cross-reactions with this antiserum were observed for all phycoerythrocyanins. 


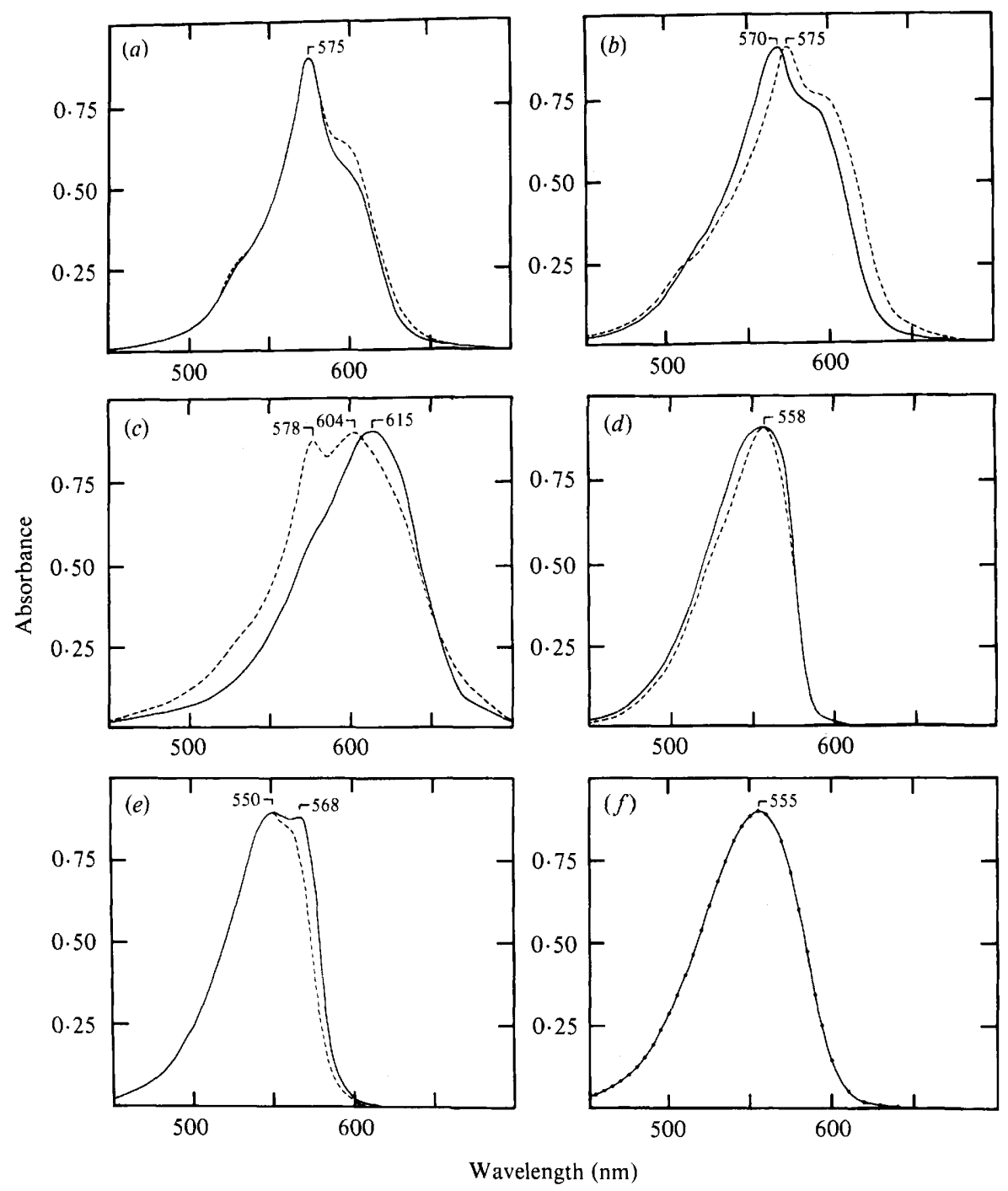

Fig. 1. Absorption spectra (in $50 \mathrm{~mm}$-sodium acetate buffer $\mathrm{pH} \mathrm{5.5)} \mathrm{of} \mathrm{cyanobacterial} \mathrm{phycoerythro-}$ cyanins and phycoerythrins, and the effect of light intensity on the phycoerythrocyanin content of Chroococcidiopsis PCC 7203. (a) Absorption spectra of Chlorogloeopsis PCC $6718(-(-)$ and PCC strain 7702 (generic assignment unknown;----) phycoerythrocyanins. (b) Absorption spectra of Scytonema PCC $7110(-)$ and Calothrix PCC $7810(---)$ phycoerythrocyanins. (c) Absorption spectra of whole cell extracts of Chroococcidiopsis PCC 7203 grown under high light intensity $\left(4.0 \times 10^{3} \mathrm{erg} \mathrm{cm}^{-2} \mathrm{~s}^{-1}\right.$; — $)$ or low light intensity $\left(1\right.$ to $2 \times 10^{2} \mathrm{erg} \mathrm{cm}^{-2} \mathrm{~s}^{-1}$; ---- ). At low light intensity the content of phycoerythrocyanin is approximately 12 -fold higher (relative to the allophycocyanin content). (d) Absorption spectra of Synechococcus PCC 7822 phycoerythrin $(-\longrightarrow)$ and of the typical C-phycoerythrin of Pseudanabaena PCC 7409 (--- ). (e) Absorption spectra of Gloeothece PCC 6909 (_- ) and Oscillatoria PCC 7823 (---) phycoerythrins. $(f)$ Absorption spectra of Pseudanabaena PCC $7409(\longrightarrow)$ and Gloeothece PCC $6909(\ldots \ldots)$ phycoerythrins in $0.8 \mathrm{M}$-urea at $\mathrm{pH} 3.0$. Identical spectra, typical of phycoerythrobilin under these conditions (Glazer \& Hixson, 1975), were also obtained with Oscillatoria PCC 7823 and Synechococcus PCC 7822 phycoerythrins.

\section{RESULTS AND DISCUSSION}

The absorption spectra of phycoerythrocyanins isolated from a wide range of cyanobacteria were remarkably similar (Figs 1 and 2). The long-wavelength absorption maxima of 


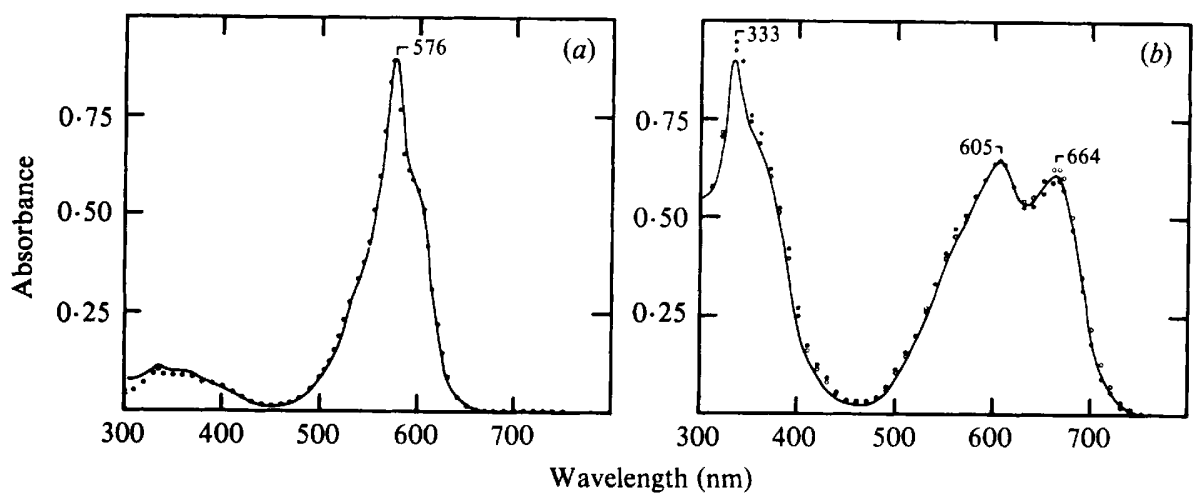

Fig. 2. Absorption spectra of phycoerythrocyanins under non-denaturing and denaturing conditions. (a) Absorption spectra of Chroococcidiopsis PCC $7203(-)$ and Fischerella PCC 7603 (= Mastigocladus laminosus Cohn; filled circles) phycoerythrocyanins in 50 mM-sodium acetate buffer $\mathrm{pH}$ 5.5. (b) Absorption spectra of Chroococcidiopsis PCC 7203 ( circles) and Calothrix PCC 7810 (filled circles) phycoerythrocyanins in $8.0 \mathrm{M}$-urea at $\mathrm{pH} 3.0$. The absorption spectra of these phycoerythrocyanins were identical to that obtained with Anabaena PCC 6411 phycoerythrocyanin under the same conditions (Bryant et al., 1976) and indicate a relative chromophore content of two phycocyanobilins per phycobiliviolin-type chromophore.

these biliproteins varied within the narrow range 568 to $577 \mathrm{~nm}$; shoulders were observed at 515 to $530 \mathrm{~nm}$ and 595 to $600 \mathrm{~nm}$ for all phycoerythrocyanins examined. However, the ratio of the absorbance at the maximum to that at the 595 to $600 \mathrm{~nm}$ shoulder varied significantly (Figs 1 and 2). As frequently only small amounts of protein were isolated, this variation may have been due to differences in the aggregation state caused by the low protein concentrations of some of the solutions. The absorption properties of biliproteins are known to vary considerably as a function of protein concentration and aggregation state (Glazer et al., 1973; Glazer \& Bryant, 1975; Glazer, 1976).

The spectroscopic properties of the chromophores of biliproteins can be studied free from the influence of the polypeptide chains when the proteins are denatured with acidic $8.0 \mathrm{M}$-urea (Glazer \& Fang, 1973; Glazer \& Hixson, 1975, 1977; Bryant et al., 1976). The chromophore compositions of the phycoerythrocyanins of three organisms (Fischerella PCC 7603, Calothrix PCC 7810, and Chroococcidiopsis PCC 7203) were examined under these denaturing conditions. As shown in Fig. $2(b)$, the absorption properties of these three proteins were identical when dissolved in $8.0 \mathrm{M}$-urea at $\mathrm{pH} \mathrm{3.0.} \mathrm{Moreover,} \mathrm{these} \mathrm{absorption} \mathrm{spectra,}$ with maxima at 333, 605 and $664 \mathrm{~nm}$, were identical to that of Anabaena PCC 6411 phycoerythrocyanin under the same conditions (Bryant et al., 1976). The phycoerythrocyanin of Anabaena PCC 6411 was shown to carry two phycocyanobilin chromophores on the $\beta$ subunit and a phycobiliviolin-type chromophore of unknown structure on the $\alpha$ subunit (Bryant et al., 1976). This chromophore distribution presumably applies to the phycoerythrocyanins described in this study as well.

All phycoerythrocyanins cross-reacted strongly with an antiserum prepared against Anabaena PCC 6411 phycoerythrocyanin (not shown). On the other hand, no phycoerythrocyanin tested gave any detectable cross-reaction with an antiserum prepared against Synechocystis PCC 6808 phycoerythrin. Thus, as found previously for other spectroscopic classes of biliproteins (Berns, 1967; Glazer et al., 1971; Bryant, 1977), the antigenic properties of phycoerythrocyanin are strongly conserved.

A wide variety of cyanobacteria (Anabaena PCC 6411, Nostoc PCC 6720, Aphanizomenon spp., Chlorogloeopsis PCC 6718, and Chroococcidiopsis PCC strains 7203, 7431, $7432,7433,7436$ and 7439 ) were tested for the ability to photocontrol phycoerthrocyanin synthesis through a complementary chromatic adaptation mechanism. No strain tested 
Table 2. Cyanohacterial strains which do not synthesize either phycoerythrin or phycoerythrocyanin

The section and generic assignments are those of Rippka et al. (1979).

Generic assignment

Synechococcus
Gloeothece
Synechocystis
Microcystis
Gloeocapsa
Chroococcidiopsis
Spirulina
Oscillatoria
Pseudanabaena
Lyngbya-Plectonema-
Phormidium group
Nodularia
Nostoc
Calothrix

Pasteur Culture Collection no.

Section I

$6301,6307,6311,6312,6603,6710,6713,6715,6716,6717,6904,6907,6908$, 6910, 6911, 7001, 7002, 7003, 7009, 7117, 7202, 73109, 7418, 7425, 7502, 7511,7809 73017

$6308,6702,6714,6803,6804,6805,6806,6902,6905,6906,7005,7008,7201$, $7509,7719,7813$

7806,7820

7428,7701

7434

Section II

Section III

6313, 7344*, 7345, 'Oswald'*

6304, 6401, 6407, 6412, 6506, 7412, 7805, 7811, 'limnetica'

$6901,6903,7402,7403,7429,7704$

$6306,6402,7004,7104,7105,7114,7123,7124,73110,7404,7406,7407,7410$,

$7411,7419,7420,7427,7505,7602,7703,7705$

73104, 7804

Section IV

7413,7423

$7102,7111,7711$

* Strains were unialgal but not axenic.

showed any significant increase in phycoerythrocyanin synthesis during growth in green light. This result differs from that of Fueglistaller et al. (1980), who recently reported that synthesis of phycoerythrocyanin in Fischerella PCC 7603 (Mastigocladus laminosus Cohn) was subject to control by a complementary chromatic adaptation mechanism.

Although phycoerythrocyanin synthesis did not appear to be photocontrolled by the wavelength of the incident light during growth, phycoerythrocyanin synthesis did appear to be dramatically affected by light intensity. As shown in Fig. 1(c), when Chroococcidiopsis PCC 7203 was grown under low light intensity, up to 40 to $50 \%$ of the biliprotein of this strain was phycoerythrocyanin. When this strain was grown under high light intensity, only small amounts of phycoerythrocyanin were synthesized. Similar but somewhat less dramatic differences were observed when other strains were grown under high and low light intensities. Thus, extracts of cells grown under low light intensity were used in screening for the synthesis of phycoerythrocyanin.

During the course of these studies, phycoerythrins with a variety of spectroscopic characteristics were detected. The most common cyanobacterial phycoerythrin spectral-form possessed a single, rather narrow absorption band with its maximum at 555 to $565 \mathrm{~nm}$ (e.g. Pseudanabaena PCC 7409 phycoerythrin, Fig. 1d). This phycoerythrin class is the typical cyanobacterial (C-type) phycoerythrin; a number of phycoerythrins of this type have been described in detail (Bennett \& Bogorad, 1971; Glazer \& Hixson, 1975; Muckle \& Rüdiger, 1977; Zickendraht-Wendelstadt et al., 1980). Other phycoerythrin spectral forms detected included those with broadened absorption bands and maxima at 550 to $565 \mathrm{~nm}$ (e.g. Synechococcus PCC 7822 phycoerythrin; Fig. 1d), and those possessing two absorption maxima or a maximum and a shoulder in the 550 to $570 \mathrm{~nm}$ region (e.g. Oscillatoria PCC 7823 and Gloeothece PCC 6909; Fig. 1e). These spectroscopic differences apparently arise from chromophore-chromophore and chromophore-protein interactions and do not reflect differences in chromophore composition. When these spectroscopically variable 
Table 3. Cyanobacterial strains which synthesize phycoerythrocyanin

The section and generic assignments are those of Rippka et al. (1979).

Generic assignment

Chroococcidiopsis

Pseudanabaena

Anabaena

Cylindrospermum

Nostoc

Aphanizomenon

Scytonema

Calothrix

Fischerella

Chlorogloeopsis

Section and generic

assignment unk known
Pasteur Culture Collection no.

Section II

7203, 7431, 7432, 7433, 7436, 7439

6406

Section III

Section IV

$6309,6411^{*}, 7108,7118^{*}, 7119,7120,7122$

$73101,7417,7604$

$6314,6705,6719,6720,7107,7416,7524$

No strain number $\dagger$

7110,7814

$7116,7204,7426,7507,7810,7815$

Section $\mathrm{V}$

$7115,73103,7414,7520,7521,7522,7523,7603 \ddagger$

6718,6912

$7517,7518,7519,7702$

* Phycoerythrocyanin purified and characterized (Bryant et al., 1976).

$\dagger$ Strain was unialgal but not axenic.

$\ddagger$ Phycoerythrocyanin purified and characterized (Fueglistaller et al., 1980; Nies \& Wehrmeyer, 1980).

phycoerythrins were denatured with $8.0 \mathrm{M}$-urea at $\mathrm{pH} 3.0$, phycoerythrobilin was the only chromophore detected (Fig. 1f). Quite different results were obtained with Gloeobacter violaceus PCC 7421 phycoerythrin. The $G$. violaceus phycoerythrin, which has absorption maxima at 501 and $564 \mathrm{~nm}$ and which carries phycourobilin and phycoerythrobilin chromophores in the ratio 1:6, was recently characterized in considerable detail (Bryant $e t$ al., 1981).

Tables 2,3 and 4 summarize a survey of the pigmentation patterns of 240 strains of cyanobacteria. In these tables, the cyanobacterial genera have been divided into five major sub-groups: Section I, unicellular cyanobacteria; Section II, baeocyte-forming cyanobacteria; Section III, filamentous, non-heterocystous cyanobacteria; Section IV, filamentous, heterocystous cyanobacteria; and Section V, heterocystous cyanobacteria which exhibit true branching (Rippka et al., 1979). The tentative generic assignments are those of Rippka et al., (1979) and Rippka \& Stanier (1979).

Several important observations can be drawn from the data of Tables 2, 3 and 4. Firstly, it should be noted that strains synthesized either phycoerythrocyanin or phycoerythrin but not both. Phycoerythrocyanin was largely found to be limited to two sub-groups of cyanobacteria, those which form heterocysts (Sections IV and V). Phycoerythrocyanin was not detected in any unicellular cyanobacterium (Section I, 66 strains examined), and only one strain, Pseudanabaena PCC 6406, of Section III (65 strains examined) contained this accessory pigment. In contrast, phycoerythrocyanin was found in 26 of the 63 strains of Section IV examined and in all 10 strains of Section $\mathrm{V}$ examined. Additionally, phycoerythrocyanin was detected in four rather similar strains whose generic assignment is uncertain. Three of these strains were received as species of Mastigocladus (synonymous with Fischerella, Section V; Rippka et al., 1979) and, although they no longer form heterocysts, may have been heterocystous at one time.

Among the strains of Section II, phycoerythrocyanin was confined to members of the genus Chroococcidiopsis, in which it occurred in six of eight strains. This finding might signal a phylogenetic relationship of these organisms to the heterocystous cyanobacteria of Sections 
Table 4. Cyanobacterial strains which synthesize phycoerythrin

\begin{abstract}
The section and generic assignments are those of Rippka et al. (1979). This table includes the data on the patterns of chromatic adaptation described by Tandeau de Marsac (1977). Symbols in parentheses following strain numbers denote the pattern of chromatic adaptation observed: (I), does not chromatically adapt; (II), chromatically adapts by photocontrolling phycoerythrin synthesis alone; (III), chromatically adapts by photocontrolling the synthesis of both phycoerythrin and phycocyanin; (ND), pattern of chromatic adaptation not determined.
\end{abstract}

Generic assignment

Synechococcus
Gloeothece
Gloeobacter
Synechocystis
Gloeocapsa
Chamaesiphon

Dermocarpa
Xenococcus
Dermocarpella
Myxosarcina
Chroococcidiopsis
Pleurocapsa group


Spirulina*
Oscillatoria
Pseudanabaena
Lyngbya-Plectonema-
Phormidium group

Nostoc

Calothrix

Section and generic assignment unknown
Pasteur Culture Collection no. and chromatic adaptation pattern

\section{Section 1}

7335 (III), 7336 (III), 7424 (I), 7822 (I)

6501 (I), 6909 (I), 7109 (I), 73108 (I)

7421 (I)

6701 (II), 6711 (II), 6808 (II)

7007 (I), 73106 (I), 7501 (I), 7512 (I)

6605 (I), 7430 (I)

Section II

7301 (I), 7302 (III), 7303 (III), 7304 (III), 7437 (III), 7438 (III)

7305 (III), 7306 (III), 7307 (III)

7326 (III)

7312 (III), 7325 (III)

6712 (III)

7310 (I), 7314 (III), 7317 (I), 7319 (III), 7320 (III), 7321 (I), 7322 (III), 7324 (I), 7327 (I), 7440 (III), 7506 (ND), 7516 (III)

Section III

7340 (ND), 7341 (ND), 7342 (ND), 7343 (ND)

6602 (I), 7112 (I), 7515 (I), 7801 (I), 7821 (I), 7823 (I)

6802 (III), 7367 (I), 7408 (III), 7409 (III)

6409 (III), 6703 (III), 7113 (II), 7375 (I), 7376 (I), 7816 (I), 7817 (I), 7818 (I), 7819 (I), 'sordida' (I)

\section{Section IV}

6302 (I), 6310 (II), 7121 (I), 73102 (II), 7422 (I), 7706 (II), 7707 (I),

7717 (III), 7718 (III), 7803 (II), 7807 (I), 7808 (I), 7812 (I),

6303 (II), 6305 (III) $\dagger, 6601$ (III) $\dagger, 7101$ (III), 7103 (III), 7415 (III),

7504 (III), 7601 (III), 7708 (II), 7709 (II), 7710 (III), 7712 (III),

7713 (III), 7714 (I), 7715 (II), 7716 (II)

7126 (III)

* These Spirulina strains were unialgal but not axenic.

† Originally reported to control phycoerythrin synthesis only, by Tandeau de Marsac (1977).

IV and V. In particular, Chroococcidiopsis strains resemble the strains of Section V in a number of ways: production of phycoerythrocyanin; mean DNA base composition (Herdman et al., 1979b); genome size (Herdman et al., 1979a); thylakoid structure (Waterbury \& Stanier, 1978); formation of an additional outer wall layer (Waterbury \& Stanier, 1978); photoheterotrophic behaviour (Ripka et al., 1979); and, finally, ability to synthesize nitrogenase under anaerobic or microaerophilic conditions (Rippka et al., 1979).

In contrast to the rather limited distribution of the accessory pigment phycoerythrocyanin, phycoerythrin was widely distributed among all cyanobacteria of Sections I-IV; at present, no phycoerythrin-containing strains exhibiting true branching exist in the Pasteur Culture Collection. Some clustering of phycoerythrin-containing strains was observed (e.g. among the genera Gloeothece, Gloeocapsa, Nostoc and Calothrix); nearly half of the strains of Section IV synthesize phycoerythrin. One important correlation of pigmentation with habitat was evident. About $80 \%$ of the strains of marine origin (Rippka et al., 1979), a grouping which includes most of the strains of Section II, synthesize either phycoerythrin or phycoerythrocyanin. These accessory pigments would be expected to confer an advantage upon those strains in natural habitats, as light of wavelengths other than those of the region 
450 to $550 \mathrm{~nm}$ are severely attenuated by columns of sea water (Ramus et al., 1976; Glazer, 1977).

The limited occurrence of the accessory pigment phycoerythrocyanin suggests that this protein might be a useful taxonomic marker. This view is supported by the results of a study of the relatedness, determined by the technique of DNA-DNA hybridization, of the cyanobacteria of Sections IV and V (Lachance, 1979, 1981). These studies indicated that the DNAs of organisms containing phycoerythrocyanin were not closely related to those of organisms containing phycoerythrin. Although the absence of both accessory pigments obviously precludes the formulation of conclusions concerning relatedness, the ability to synthesize phycoerythrin or phycoerythrocyanin might be useful as an exclusionary character in the identification of certain strain clusters of heterocystous cyanobacteria.

Table 4 includes the results of the present study, as well as those of Tandeau de Marsac (1977), concerning the patterns of chromatic adaptation exhibited by the cyanobacteria. As found for phycoerythrin synthesis, ability to photocontrol phycoerythrin (or phycoerythrin and phycocyanin) synthesis is a widely distributed trait. For example, among the closely related strains of a single Calothrix cluster (Lachance, 1979, 1981), all possible patterns of chromatic adaptation were found (phycoerythrin absent; phycoerythrin present but not photocontrolled; phycoerythrin present and photocontrolled; phycoerythrin and phycocyanin photocontrolled). At present, the ability to exhibit chromatic adaptation does not appear to be a very useful marker for cyanobacterial taxonomy.

The author was supported by a post-doctoral fellowship awarded in an exchange programme of the National Science Foundation and the Centre National de la Recherche Scientifique and gratefully acknowledges the hospitality of the Unite de Physiologie Microbienne, Institut Pasteur. Thanks are extended to T. Coursin, A. M. Castets, G. Guglielmi and R. Rippka for assistance in growing the cultures used in this work; to G. Cohen-Bazire, M. A. Lachance, N. Tandeau de Marsac, R. Rippka and R. Y. Stanier for helpful discussions; and to G. Guglielmi for assistance in the preparation of the figures. This research was supported by grants from the Centre National de la Recherche Scientifique (E.R.A. 398) and the Délégation Générale à la Recherche Scientifique et Technique (Décision d'aide no. 77-7-1751).

\section{REFERENCES}

Bennett, A. \& Bogorad, L. (1971). Properties of subunits and aggregates of blue-green algal biliproteins. Biochemistry 10, 3625-3634.

BERNS, D. S. (1967). Immunochemistry of biliproteins. Plant Physiology 42, 1569-1586.

Bogorad, L. (1975). Phycobiliproteins and complementary chromatic adaptation. Annual Review of Plant Physiology 26, 369-401.

BryanT, D. A. (1977). Comparative studies on cyanobacterial and rhodophytan biliproteins. Ph.D. thesis, University of California, Los Angeles.

Bryant, D. A., Glazer, A. N. \& Eiserling, F. A. (1976). Characterization and structural properties of the major biliproteins of Anabaena sp. Archives of Microbiology 110, 61-75.

Bryant, D. A., Hixson, C. S. \& Glazer, A. N. (1978). Structural studies on phycobiliproteins. III. Comparison of bilin-containing peptides from the $\beta$ subunits of C-phycocyanin, R-phycocyanin, and phycoerythrocyanin. Journal of Biological Chemistry 253, 220-225.

Bryant, D. A., Guglielmi, G., Tandeau DE Marsac, N., Castets, A. M. \& Cohen-Bazire, G. (1979). The structure of cyanobacterial phycobilisomes: a model. Archives of Microbiology $123,113-127$.
Bryant, D. A., Cohen-Bazire, G. \& Glazer, A. N. (1981). Characterization of the biliproteins of Gloeobacter violaceus. Archives of Microbiology 129, 190-198.

Cohen-Bazire, G., Beguin, S., Rimon, S., Glazer, A. N. \& Brown, D. M. (1977). Physicochemical and immunological properties of allophycocyanins. Archives of Microbiology 111, 225-238.

Fueglistaller, P., Widmer, H., Sidler, W., Frank, G. \& ZUBER, H. (1980). Chromatic adaptation and phycoerythrocyanin in the thermophilic cyanobacterium Mastigocladus laminosus. Fifth International Congress on Photosynthesis, Halkidiki, Greece, Abstracts, 192.

GANTT, E. (1980). Structure and function of phycobilisomes: light harvesting pigment complexes in red and blue-green algae. International Review of Cytology 66, 45-80.

Glazer, A. N. (1976). Phycocyanins: structure and function. Photochemical and Photobiological Reviews 1, 71-115.

Glazer, A. N. (1977). Structure and molecular organization of the photosynthetic accessory pigments of cyanobacteria and red algae. Molecular and Cellular Biochemistry 18, 125-140.

Glazer, A. N. \& Bryant, D. A. (1975). Allo- 
phycocyanin B $\left(\lambda_{\max } 671,618 \mathrm{~mm}\right)$ : a new cyanobacterial phycobiliprotein. Archives of Microbiology 104, 15-22.

Glazer, A. N. \& FANG, S. (1973). Chromophore content of blue-green algal phycobiliproteins. Journal of Biological Chemistry 248, 659-662.

Glazer, A. N. \& Hixson, C. S. (1975). Characterization of R-phycocyanin. Chromophore content of R-phycocyanin and C-phycoerythrin. Journal of Biological Chemistry 250, 5487-5495.

Glazer, A. N. \& Hixson, C. S. (1977). Subunit structure and chromophore composition of rhodophytan phycoerythrins. Porphyridium cruentum B-phycoerythrin and b-phycoerythrin. Journal of Biological Chemistry 252, 32-42.

Glazer, A. N., Cohen-Bazire, G. \& Stanier, R. Y. (1971). Comparative immunology of algal biliproteins. Proceedings of the National Academy of Sciences of the United States of America 68, 3005-3008.

Glazer, A. N., Fang, S. \& Brown, D. M. (1973). Spectroscopic properties of C-phycocyanin and of its $\alpha$ and $\beta$ subunits. Journal of Biological Chemistry 248, 5679-5685.

Herdman, M., Janvier, M., Rippka, R. \& Stanier, R. Y. $(1979 a)$. Genome size of cyanobacteria. Journal of General Microbiology 111, 73-85.

Herdman, M., Janvier, M., Waterbury, J. B., RiPPKA, R., Stanier, R. Y. \& MANDEL, M. $(1979 b)$. Deoxyribonucleic acid base composition of cyanobacteria. Journal of General Microbiology 111, 63-71.

LACHANCE, M. A. (1979). DNA relatedness among heterocystous cyanobacteria. III International Symposium on Photosynthetic Prokaryotes, Oxford, England, Abstracts, E21.

LACHANCE, M. A. (1981). Genetic relatedness of heterocystous cyanobacteria by deoxyribonucleic acid-deoxyribonucleic acid reassociation. International Journal of Systematic Bacteriology 31, 139-147.

Ley, A. C., Butler, W. L., Bryant, D. A. \& Glazer, A. N. (1977). Isolation and function of allophycocyanin B of Porphyridium cruentum. Plant Physiology 59, 974-980.

MuCKLE, G. \& Rüdiger, W. (1977). Chromophore content of $\mathrm{C}$-phycoerythrin from various cyanobacteria. Zeitschrift für Naturforschung 32c, 957962.
NiEs, M. \& WeHRMeyer, W. (1980). Isolation and biliprotein characterization of phycobilisomes from the thermophilic cyanobacterium Mastigocladus laminosus Cohn. Planta 150, 330-337.

Porter, G., Tredwell, C. J., Searle, G. F. W. \& BARBer, J. (1978). Picosecond time-resolved energy transfer in Porphyridium cruentum. Part I. In the intact alga. Biochimica et biophysica acta 501, 232-245.

Ramus, J., Beale, S. I., Mauzerall, D. \& Howard, K. L. (1976). Changes in photosynthetic pigment concentration in seaweeds as a function of water depth. Marine Biology 37, 223-229.

RIPPKA, R. \& STANIER, R. Y. (1979). Observations on unpublished strains in the Pasteur Culture Collection of Cyanobacteria. III International Symposium on Photosynthetic Prokaryotes, Oxford, England, Abstracts, A26.

RippKa, R., Deruelles, J., Waterbury, J. B., Herdman, M. \& Stanier, R. Y. (1979). Generic assignments, strain histories, and properties of pure cultures of cyanobacteria. Journal of General Microbiology 111, 1-61.

Searle, G. F. W., Barber, J., Porter, G. \& Tredwell, C. J. (1978). Picosecond time-resolved energy transfer in Porphyridium cruentum. Part II. In the isolated light harvesting complex (phycobilisomes). Biochimica et biophysica acta 501, 246-256.

Stanier, R. Y. \& Cohen-Bazire, G. (1977). Phototrophic prokaryotes: the cyanobacteria. Annual Review of Microbiology 31, 225-274.

Stanier, R. Y., Kunisawa, R., Mandel, M. \& Cohen-Bazire, G. (1971). Purification and properties of unicellular blue-green algae (Order Chroococcales). Bacteriological Reviews 35, 171-205.

TANDEAU DE MARSAC, N. (1977). Occurrence and nature of chromatic adaptation in cyanobacteria. Journal of Bacteriology 130, 82-91.

Waterbury, J. B. \& Stanier, R. Y. (1978). Patterns of growth and development in pleurocapsalean cyanobacteria. Microbiological Reviews 42, 2-44.

ZickendRaht-WendelstadT, B., Friedrich, J. \& RÜDIGER, W. (1980). Spectral characterization of monomeric C-phycoerythrin from Pseudanabaena $\mathrm{W} 1173$ and its $\alpha$ and $\beta$ subunits: energy transfer in isolated subunits and C-phycoerythrin. Photochemistry and Photobiology 31, 367-376. 